\title{
Inner Symmetries for Moebius Maps
}

\author{
By \\ Fritz Schweiger \\ (Vorgelegt in der Sitzung der math.-nat. Klasse am 11. Dezember 2008 \\ durch das k. M. I. Fritz Schweiger)
}

\begin{abstract}
Differentiable isomorphisms of Moebius systems are considered (SCHWEIGER [2]). A map is called an inner symmetry if it commutes with the map Tof the Moebius system and permutes the cells of the time-1-partition. This notion is discussed for Moebius systems with two and three branches. An extension to 2-dimensional cases is outlined.
\end{abstract}

Mathematics Subject Classification (2000): 11K55, 28 D99.

Key words: Ergodic theory, invariant measures.

\section{Introduction}

Definition. Let $B$ be an interval and $T: B \rightarrow B$ be a map. We assume that there is a countable collection of intervals $\left(J_{k}\right), k \in I, \# I \geq 2$ and an associated sequence of matrices

$$
\alpha(k)=\left(\begin{array}{ll}
a_{k} & b_{k} \\
c_{k} & d_{k}
\end{array}\right),
$$

$a_{k} d_{k}-b_{k} c_{k} \neq 0$, with the properties:

- $\bigcup_{k \in I} \overline{J_{k}}=\bar{B}, J_{m} \cap J_{n}=\emptyset$ if $n \neq m$.

- $T x=\frac{c_{k}+d_{k} x}{a_{k}+b_{k} x}, \quad x \in J_{k}$.

- $\left.T\right|_{J_{k}}$ is a bijective map from $J_{k}$ onto $B$.

Then we call $(B, T)$ a Moebius system. 
This is a special case of a fibred system (SCHWEIGER [1]). Since $\left.T\right|_{J_{k}}$ is bijective the inverse map $V_{k}: B \rightarrow J_{k}$ exists. The corresponding matrix will be denoted by $\beta(k)$. We denote furthermore

$$
\omega(k ; x):=\left|V_{k}^{\prime}(x)\right|=\frac{\left|a_{k} d_{k}-b_{k} c_{k}\right|}{\left(d_{k}-b_{k} x\right)^{2}} .
$$

Then a nonnegative measurable function $h$ is the density of an invariant measure iff $h(x)=\sum_{k \in I} h\left(V_{k} x\right) \omega(k ; x)$.

Remark. It is easy to see that we can assume $B=[a, b], B=[a, \infty[$ or $B=]-\infty, b]$ but $B=\mathbb{R}$ is excluded (since $\# I \geq 2$ ).

Definition. The Moebius system $\left(B^{*}, T^{*}\right)$ is called a natural dual of $(B, T)$ if there is a partition $\left\{I_{k}^{*}\right\}$ such that

$$
T^{*} y=\frac{b_{k}+d_{k} y}{a_{k}+c_{k} y},
$$

i.e., the matrix $\alpha^{*}(k)$ is the transposed matrix of $\alpha(k)$.

In the paper SCHWEIGER [2] the following definition was given.

Definition. The Moebius system $\left(B^{*}, T^{*}\right)$ is differentiably isomorphic to $(B, T)$ if there is a map $\psi: B \rightarrow B^{*}$ such that $\psi^{\prime}$ exists almost everywhere and the commutativity condition $\psi \circ T=T^{*} \circ \psi$ holds.

What I had in mind was a more precise definition, namely for all $k \in I$ the commutativity condition

$$
\psi \circ \alpha(k)=\alpha(k)^{*} \circ \psi
$$

should hold and this property was used in all what followed in the paper. However, one might ask if it is possible that

$$
\psi \circ \alpha(k)=\alpha(\pi k)^{*} \circ \psi
$$

holds for a permutation $\pi: I \rightarrow I$ of the index set.

\section{The Case of Two Branches}

It is well known that in the case of two branches, namely $\# I=2$ the system $\left(B^{*}, T^{*}\right)$ is always differentiably isomorphic to $(B, T)$ in the sense of Eq. (1). We take $B=[0,1]$ with $c=\frac{1}{2}$ as the midpoint of 
the partition. We put $I=\{\lambda, \mu\}$ and there are four subtypes $\left(\varepsilon_{1}, \varepsilon_{2}\right)$ where $\varepsilon_{j}=1$ stands for an increasing map and $\varepsilon_{j}=-1$ for a decreasing map. Now we ask for the possibility of satisfying (2) with exchanging $\lambda$ and $\mu$

$$
\begin{aligned}
& \varphi \circ \alpha(\lambda)=\alpha(\mu)^{*} \circ \varphi, \\
& \varphi \circ \alpha(\mu)=\alpha(\lambda)^{*} \circ \varphi .
\end{aligned}
$$

Since $\psi \circ \alpha(k)=\alpha(k)^{*} \circ \psi, k \in\{\lambda, \mu\}$, is always satisfied, we obtain

$$
\psi^{-1} \circ \varphi \circ \alpha(\lambda)=\psi^{-1} \circ \alpha(\mu)^{*} \circ \varphi=\alpha(\mu) \circ \psi^{-1} \circ \varphi .
$$

Therefore the system $(B, T)$ allows an inner symmetry $\chi=\psi^{-1} \circ \varphi$ such that

$$
\chi \circ V(\lambda)=V(\mu) \circ \chi .
$$

Conversely, if $(B, T)$ allows such an inner symmetry, we can find a differentiable map $\varphi$ which satisfies $(3)$, namely $\varphi=\psi \circ \chi$. Therefore it is enough to solve Eq. (4).

Theorem 1. (a) For types $(1,1)$ and $(-1,-1)$ there are infinitely many solutions of Eq. (4).

(b) For types $(1,-1)$ and $(-1,1)$ no solution exists.

Proof. The only Moebius transformation which permutates the points $\left\{0, \frac{1}{2}, 1\right\}$ in an appropriate way is $\chi(t)=1-t$.

It is easy to see that the matrices $\alpha(\lambda)$ and $\alpha(\mu)$ depend on one free parameter. The conditions on $\lambda$ and $\mu$ are necessary to avoid attractive fixed points or poles in the domain of definition.

(a) Type $(1,1), 0<\lambda \leq 1, \mu \leq 0$ :

$$
\begin{aligned}
\alpha(\lambda)=\left(\begin{array}{cc}
\lambda & 1-2 \lambda \\
0 & 1
\end{array}\right), \quad \alpha(\mu) & =\left(\begin{array}{cc}
\mu & -1-\mu \\
1 & -2
\end{array}\right)\left(\begin{array}{cc}
1 & 0 \\
1 & -1
\end{array}\right)\left(\begin{array}{cc}
\lambda & 1-2 \lambda \\
0 & 1
\end{array}\right) \\
& =\rho\left(\begin{array}{cc}
\mu & -1-\mu \\
1 & -2
\end{array}\right)\left(\begin{array}{cc}
1 & 0 \\
1 & -1
\end{array}\right) .
\end{aligned}
$$

From this we see

$$
\lambda=\frac{1}{1-\mu}=-\rho .
$$

Note that $0<\lambda \leq 1$ is consistent with $\mu \leq 0$. 
Type $(-1,-1), \lambda<2, \mu<2$ :

$$
\begin{aligned}
\alpha(\lambda)=\left(\begin{array}{cc}
1 & -\lambda \\
1 & -2
\end{array}\right), \quad \alpha(\mu) & =\left(\begin{array}{cc}
\mu & 2-2 \mu \\
2 & -2
\end{array}\right)\left(\begin{array}{cc}
1 & 0 \\
1 & -1
\end{array}\right)\left(\begin{array}{cc}
1 & -\lambda \\
1 & -2
\end{array}\right) \\
& =\rho\left(\begin{array}{cc}
\mu & 2-2 \mu \\
2 & -2
\end{array}\right)\left(\begin{array}{cc}
1 & 0 \\
1 & -1
\end{array}\right) .
\end{aligned}
$$

This gives the conditions

$$
\begin{aligned}
\rho & =\frac{1}{2-\mu}, \\
\lambda & =\frac{2-2 \mu}{2-\mu} .
\end{aligned}
$$

(b) For type $(1,-1)$ the branch $\alpha(\lambda)$ has the fixed point $x=0$ but $x=1$ is not a fixed point for $\alpha(\mu)$. A similar reasoning excludes the type $(-1,1)$.

\section{The Case of Three Branches}

As in SCHWEIGER [2] we consider the partition $0<\frac{1}{2}<\frac{2}{3}<1$ and three maps depending on parameters $\lambda, \mu, \nu$, say. By continuity reasons the only case of satisfying (2) with a proper permutation is the exchange of $\lambda$ and $\nu$ which leaves $\mu$ fixed,

$$
\begin{aligned}
& \psi \circ \alpha(\lambda)=\alpha(\nu)^{\#} \circ \psi, \\
& \psi \circ \alpha(\mu)=\alpha(\mu)^{\#} \circ \psi .
\end{aligned}
$$

In a similar way we can also define an inner symmetry $\chi$ as a Moebius map such that

$$
\begin{aligned}
& \chi \circ \alpha(\lambda)=\alpha(\nu) \circ \chi, \\
& \chi \circ \alpha(\mu)=\alpha(\mu) \circ \chi .
\end{aligned}
$$

We will consider inner symmetries first. We will see that for three branches an inner symmetry in almost all cases leads to a solution of $(5)$.

The only Moebius transformation which exchanges $\left\{0, \frac{1}{2}, \frac{2}{3}, 1\right\}$ is

$$
\chi(t)=\frac{2-2 t}{2-t} .
$$

Looking at the cases in which either $x=0$ or $x=1$ is a fixed point we can exclude the types $(1,1,-1),(1,-1,-1),(-1,1,1)$, and $(-1,-1,1)$. For the remaining types we find the following result. 
Theorem 2. All types $(1,1,1),(1,-1,1),(-1,1,-1),(-1,-1,-1)$ allow an inner symmetry for an infinite set of parameters $\lambda$ and $\nu$.

Proof. We first list the six matrices $\alpha(k), k=\lambda, \mu, \nu$, which will be used in our calculations.

$$
\begin{aligned}
& \varepsilon_{1}=1,\left(\begin{array}{cc}
\lambda & 1-2 \lambda \\
0 & 1
\end{array}\right), \\
& \varepsilon_{1}=-1,\left(\begin{array}{cc}
-1 & -\lambda+2 \\
-1 & 2
\end{array}\right), \\
& \varepsilon_{2}=1,\left(\begin{array}{cc}
2 \mu-1 & 2-3 \mu \\
-1 & 2
\end{array}\right), \\
& \varepsilon_{2}=-1,\left(\begin{array}{cc}
\mu-2 & 3-2 \mu \\
-2 & 3
\end{array}\right), \\
& \varepsilon_{3}=1,\left(\begin{array}{cc}
\nu-2 & -\nu+3 \\
-2 & 3
\end{array}\right), \\
& \varepsilon_{3}=-1,\left(\begin{array}{cc}
2 \nu-1 & 1-3 \nu \\
-1 & 1
\end{array}\right) .
\end{aligned}
$$

(a) Type $(1,1,1), 0<\lambda \leq 1,0<\mu, 1 \leq \nu$. We consider first the equation $\chi \circ \alpha(\mu)=\alpha(\mu) \circ \chi$.

$$
\left(\begin{array}{ll}
2 & -1 \\
2 & -2
\end{array}\right)\left(\begin{array}{cc}
2 \mu-1 & 2-3 \mu \\
-1 & 2
\end{array}\right)=\left(\begin{array}{cc}
2 \mu-1 & 2-3 \mu \\
-1 & 2
\end{array}\right)\left(\begin{array}{cc}
2 & -1 \\
2 & -2
\end{array}\right) .
$$

This gives $\mu=\frac{1}{2}$ as the unique solution for the middle branch. The further equations

$$
\left(\begin{array}{ll}
2 & -1 \\
2 & -2
\end{array}\right)\left(\begin{array}{cc}
\lambda & 1-2 \lambda \\
0 & 1
\end{array}\right)=\rho\left(\begin{array}{cc}
\nu-2 & -\nu+3 \\
-2 & 3
\end{array}\right)\left(\begin{array}{ll}
2 & -1 \\
2 & -2
\end{array}\right)
$$

give the relation $\lambda \nu=1$ which is compatible with the results in SCHWEIGER [2].

(b) Type $(-1,1,-1), 0<\lambda, 0<\mu, 0<\nu$. In a similar way we find from

$$
\left(\begin{array}{ll}
2 & -1 \\
2 & -2
\end{array}\right)\left(\begin{array}{cc}
-1 & -\lambda+2 \\
-1 & 2
\end{array}\right)=\rho\left(\begin{array}{cc}
2 \nu-1 & 1-3 \nu \\
-1 & 1
\end{array}\right)\left(\begin{array}{ll}
2 & -1 \\
2 & -2
\end{array}\right)
$$

the relation $4 \lambda \nu=1$. 
(c) Type $(1,-1,1), 0<\lambda \leq 1,0<\mu, 1 \leq \nu$. We need only consider the equation $\chi \circ \alpha(\mu)=\alpha(\mu) \circ \chi$,

$$
\left(\begin{array}{ll}
2 & -1 \\
2 & -2
\end{array}\right)\left(\begin{array}{cc}
\mu-2 & 3-2 \mu \\
-2 & 3
\end{array}\right)=\left(\begin{array}{cc}
\mu-2 & 3-2 \mu \\
-2 & 3
\end{array}\right)\left(\begin{array}{cc}
2 & -1 \\
2 & -2
\end{array}\right) .
$$

This gives the unique value $\mu=1$. As before the other equations show $\lambda \nu=1$.

(d) Type $(-1,-1,-1), 0<\lambda, 0<\mu, 0<\nu$. This gives the conditions $\mu=1$ and $4 \lambda \nu=1$.

Remark. The invariant density $h=h(x)$ satisfies the equation $h(x)=h(\chi(x))\left|\chi^{\prime}(x)\right|$. If $\xi_{\lambda}, \xi_{\mu}, \xi_{\nu}$ are the fixed points of $T$ then clearly we find $\chi\left(\xi_{\lambda}\right)=\xi_{\nu}, \chi\left(\xi_{\mu}\right)=\xi_{\mu}$, and $\chi\left(\xi_{\nu}\right)=\xi_{\lambda}$.

Theorem 3. (a) For type $(1,1,1)$ there is no solution of Eq. (2).

(b) For types $(1,-1,1),(-1,1,-1),(-1,-1,-1)$ there are infinitely many solutions.

Proof. Type $(1,1,1)$ : For the existence of an inner symmetry we found the relation $\lambda \nu=1$ and $\mu=\frac{1}{2}$. A differentiably isomorphic dual (Eq. (1)) exists if the condition

$$
2 \lambda \mu+2 \nu=\lambda \nu+\lambda
$$

is satisfied. This leads to $2 \nu=1$ which is not allowed.

Type $(1,-1,1)$ : Here we found $\lambda \nu=1$ and $\mu=1$. A differentially isomorphic dual exists if the condition

$$
\lambda \nu=\mu
$$

holds. Therefore any inner symmetry leads to a solution of (5) but not vice versa.

Type $(-1,1,-1)$ : Here we had $\mu=\frac{1}{2}$ and $4 \lambda \nu=1$. The condition for an isomorphic dual is given as

$$
2 \lambda \mu+\mu=2 \lambda \nu+\lambda
$$

which is satisfied.

Type $(-1,-1,-1)$ : In this case $\mu=1$ and $4 \lambda \nu=1$. The condition

$$
4 \lambda \nu+\lambda+\nu=\mu \nu+\lambda \mu+\mu
$$

is also satisfied. 


\section{Outlook: 2-Dimensional Generalizations}

These ideas can be extended to higher dimensions. We will consider three 2-dimensional algorithms. The kernel

$$
K\left(x_{1}, x_{2}, y_{1}, y_{2}\right)=\frac{1}{\left(1+x_{1} y_{1}+x_{2} y_{2}\right)^{3}}
$$

leads to a dual system with transposed matrices.

Brun Algorithm. We consider the map on $B=\left\{\left(x_{1}, x_{2}\right): 0 \leq x_{2} \leq\right.$ $\left.x_{1} \leq 1\right\}$

$$
T\left(x_{1}, x_{2}\right)= \begin{cases}\left(\frac{x_{1}}{1-x_{1}}, \frac{x_{2}}{1-x_{1}}\right), & x_{1} \leq \frac{1}{2}, \\ \left(\frac{1-x_{1}}{x_{1}}, \frac{x_{2}}{x_{1}}\right), & x_{1}+x_{2} \leq 1, \\ \left(\frac{x_{2}}{x_{1}}, \frac{1-x_{1}}{x_{1}}\right), & 1 \leq x_{1}+x_{2} .\end{cases}
$$

Then the dual algorithm $T^{*}$ exists on the set $B^{*}=\left\{\left(y_{1}, y_{2}\right): 0 \leq y_{1}\right.$, $\left.0 \leq y_{2} \leq 1\right\}$. The map

$$
\psi(s, t)=\left(\frac{1-s}{s}, \frac{t}{s}\right)
$$

gives a differentiable isomorphism. Since the point $x=(0,0)$ is the only fixed point on the boundary it is seen quite easily that no inner symmetry can be found.

Selmer Algorithm. Here we consider the set $D=\left\{\left(x_{1}, x_{2}\right): 1 \leq\right.$ $\left.x_{1}+x_{2}, 0 \leq x_{2} \leq x_{1} \leq 1\right\}$ and the map

$$
T\left(x_{1}, x_{2}\right)= \begin{cases}\left(\frac{1-x_{2}}{x_{1}}, \frac{x_{2}}{x_{1}}\right), & x_{2} \leq \frac{1}{2}, \\ \left(\frac{x_{2}}{x_{1}}, \frac{1-x_{2}}{x_{1}}\right), & \frac{1}{2} \leq x_{2} .\end{cases}
$$

The dual exists on the set $D^{*}=\left\{\left(y_{1}, y_{2}\right): 0 \leq y_{1}, 0 \leq y_{2}\right\}$ and the map

$$
\psi(s, t)=\left(\frac{-1+s+t}{1-s}, \frac{s-t}{1-s}\right)
$$

is a differentiable isomorphism. Again, since $x=(1,0)$ is the only fixed point on the boundary an inner symmetry is not allowed. 
Parry-Daniels Map. We use the projected version on the triangle $\Delta=\left\{\left(x_{0}, x_{1}\right): 0 \leq x_{0}, 0 \leq x_{1}, x_{0}+x_{1} \leq 1\right\}$. The partition is labelled by permutations on the indices $0,1,2$ and the map is given piecewise as follows,

$$
\begin{aligned}
T_{\varepsilon}\left(x_{0}, x_{1}\right) & =\left(\frac{x_{0}}{1-x_{0}-x_{1}}, \frac{-x_{0}+x_{1}}{1-x_{0}-x_{1}}\right), \\
T_{(01)}\left(x_{0}, x_{1}\right) & =\left(\frac{x_{1}}{1-x_{0}-x_{1}}, \frac{x_{0}-x_{1}}{1-x_{0}-x_{1}}\right), \\
T_{(12)}\left(x_{0}, x_{1}\right) & =\left(\frac{x_{0}}{x_{1}}, \frac{1-2 x_{0}-x_{1}}{x_{1}}\right), \\
T_{(021)}\left(x_{0}, x_{1}\right) & =\left(\frac{1-x_{0}-x_{1}}{x_{1}}, \frac{-1+2 x_{0}+x_{1}}{x_{1}}\right), \\
T_{(02)}\left(x_{0}, x_{1}\right) & =\left(\frac{1-x_{0}-x_{1}}{x_{0}}, \frac{-1+2 x_{0}+x_{1}}{x_{0}}\right), \\
T_{(012)}\left(x_{0}, x_{1}\right) & =\left(\frac{x_{1}}{x_{0}}, \frac{1-x_{0}-2 x_{1}}{x_{0}}\right) .
\end{aligned}
$$

There also exists a natural dual on the set

$$
\Delta^{*}=\left\{\left(y_{1}, y_{2}\right): 0 \leq y_{2} \leq y_{1}\right\},
$$

but the systems $(\Delta, T)$ and $\left(\Delta^{*}, T^{*}\right)$ are not differentiably isomorphic in the sense of Eq. (1). To say it more precisely, no fractional linear map $\psi$ with this property exists.

However, $\left(\Delta^{*}, T^{*}\right)$ is an exceptional dual as defined in SCHWEIGER [2]. The map

$$
\psi(s, t)=\left(\frac{1}{s+t}, \frac{t}{s+t}\right)
$$

has the property

$$
\alpha(k) \circ \psi=\psi \circ \alpha^{*}(k)
$$

for all $k=\varepsilon,(02),(012),(021)$ but

$$
\begin{aligned}
& \alpha(01) \circ \psi=\psi \circ \alpha^{*}(12), \\
& \alpha(12) \circ \psi=\psi \circ \alpha^{*}(01) .
\end{aligned}
$$

This is reasonable since a closer inspection shows that the two maps $\alpha^{*}(\varepsilon)$ and $\alpha^{*}(12)$ lead to the same type of exceptional set as $\alpha(\varepsilon)$ and $\alpha(01)$ do for the map $T$. 


\section{References}

[1] SCHWEIGER, F. (2000) Multidimensional Continued Fractions. Oxford University Press, Oxford

[2] SCHWEIGER, F. (2006) Differentiable equivalence of fractional linear maps. IMS Lecture Notes-Monograph Series Dynamics \& Stochastics 48: 237-247

Author's address: Prof. Dr. Fritz Schweiger, Department of Mathematics, University of Salzburg, Hellbrunner Straße 34, 5020 Salzburg, Austria. E-Mail: fritz.schweiger@sbg.ac.at. 
